\title{
Generalization of the Pseudo Diagonalization Method
}

\author{
Hee-Hyol Lee $^{\dagger}$, Michio Miyazaki ${ }^{\dagger \dagger}$ and Kageo Akizuki ${ }^{\dagger \dagger}$ \\ ${ }^{\dagger}$ Fukuoka Inst. of Tech., College of Computer Engineering, lee@fit.ac.jp \\ 3-30-1, Wajirohigashi, Higashi-ku, Fukuoka, 811-0295 JAPAN \\ ${ }^{\dagger \dagger}$ Kanto Gakuin University, College of Engineering \\ 4834, Matsumure-cho, Kanazawa-ku, Yokohama, 236-8501 JAPAN \\ ${ }^{\dagger \dagger \dagger}$ Waseda University, College of Engineering \\ 3-4-1, Ohkubo, Shinjuku-ku, Tokyo, 169-8555 JAPAN
}

\begin{abstract}
The inverse Nyquist array method is one of the practical design methods in frequency region for the multi-input and multi-output system. In this method, the design of the noninteracting precompensator is reduced to the eigenvalue problem by the pseudo diagonalization method. However, the pseudo diagonalization method is applicable only to the system where numbers of input and output are equal. In this paper, we generalize the pseudo diagonalization method to be applicable to the system where numbers of input and output are not always equal.
\end{abstract}

\section{Introduction}

In multivariable control systems, controlled variables interact on each other in general. In this case, a compensation for a control loop exerts harmful influence upon other control loop through the interaction. If we could take away this interaction by some ways for a complete one to one correspondence between the manipulated variable and the controlled variable, then the design of control systems results in the individual compensation of the single loop control.

One of the practical design methods in frequency region for multi-input and multi-output systems is the inverse Nyquist array method [1] [4]. In this method, the design of the noninteracting precompensator is resulted in the eigenvalue problem by the pseudo diagonalization method [3]. However, the pseudo diagonalization method is applicable only to the system where numbers of input and output are equal. This paper proposes a generalization of the pseudo diagonalization method to be applicable to the system where numbers of input and output are not always equal, and confirms the effectiveness of the proposed method through some simulations.

\section{Generalization of the pseudo diagonalization method}

For multi-input and multi-output control systems, a controlled system $G p(s) \in C^{m x r}$, where the numbers of input $r$ and output $m$ are not always equal, a reference input $r \in R^{m x I}$, which dimension is same as the order of output $m$, and a main controller $K(s) \in C^{m x m}$, which is a diagonal matrix, are connected illustrated in Fig.1.

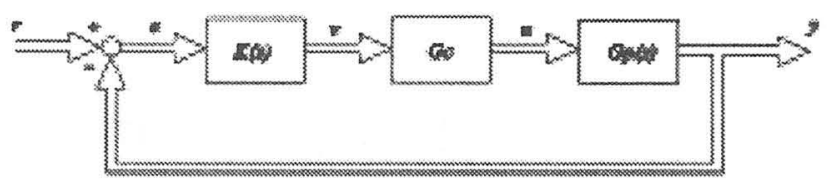

Fig.1. Multi-input and multi-output control system with a noninteracting precompensator

Let us consider the design of a precompensator $G c \in R^{r x m}$ at frequency $s=j \omega_{0}$, 


$$
G c=\left[\begin{array}{cccccc}
h_{11} & h_{12} & \cdots & h_{1 p} & \cdots & h_{1 m} \\
h_{21} & h_{22} & \cdots & h_{2 p} & \cdots & h_{2 m} \\
\vdots & \vdots & & \vdots & & \vdots \\
h_{r 1} & h_{r 2} & \cdots & h_{r p} & \cdots & h_{m m}
\end{array}\right]
$$

Then the $i j$ th element $\ell_{i j}\left(j \omega_{0}\right)$ of the transfer function matrix $G(s)=G p(s) G c \in C^{m x m}$ is represented by

$$
\ell_{i j}\left(j \omega_{0}\right)=\sum_{k=l}^{r} g_{i k}\left(j \omega_{0}\right) h_{k j}
$$

at $s=j \omega_{0}$, where $g_{i k}\left(j \omega_{0}\right)$ is the $i k$ th element of the controlled system $G p(s)$. Here the elements of the $p$ th column vector $h_{p}=\left(h_{l p}, h_{2 p}, \ldots, h_{r p}\right)^{T}$ of the precompensator $G c$ are selected so that the square-sum of the absolute value of the non-diagonal elements in the $p$ th column vector of $G\left(j \omega_{0}\right)$,

$$
\sum_{i=l, i \neq p}^{m}\left|\ell_{i p}\left(j \omega_{0}\right)\right|^{2}
$$

is minimized under condition of the constraint,

$$
h_{1 p}^{2}+h_{2 p}^{2}+\cdots+h_{r p}^{2}=1
$$

For this optimization, we obtain the extreme value of the function

$$
\begin{aligned}
\phi_{p} & =\sum_{i=1, i \neq p}^{m}\left|\ell_{i p}\left(j \omega_{0}\right)\right|^{2}+\lambda\left(1-\sum_{k=1}^{r} h_{k p}^{2}\right) \\
& =\sum_{i=l, i \neq p}^{m}\left|\sum_{k=1}^{r} g_{i k}\left(j \omega_{0}\right) h_{k p}\right|^{2}+\lambda\left(1-\sum_{k=1}^{r} h_{k p}^{2}\right)
\end{aligned}
$$

where $\lambda$ is the Lagrange multiplier.

$$
\begin{aligned}
& \text { Let } g_{i k}\left(j \omega_{0}\right)=\alpha_{i k}+j \beta_{i k} \text {, then } \\
& \begin{aligned}
\phi_{p} & =\sum_{i=l, i \neq p}^{m}\left|\sum_{k=I}^{r} \alpha_{i k} h_{k p}+j \sum_{k=l}^{r} \beta_{i k} h_{k p}\right|^{2}+\lambda\left(1-\sum_{k=1}^{r} h_{k p}^{2}\right) \\
= & \sum_{i=l, i \neq p}^{m}\left[\left(\sum_{k=I}^{r} \alpha_{i k} h_{k p}\right)^{2}+\left(\sum_{k=I}^{r} \beta_{i k} h_{k p}\right)^{2}\right]+\lambda\left(1-\sum_{k=I}^{r} h_{k p}^{2}\right)
\end{aligned}
\end{aligned}
$$

Differentiating $\phi_{p}$ with respect to $h_{j p}$ and equalizing it to zero, we can get

$$
\sum_{i=l, i \neq p}^{m}\left[\left(\sum_{k=I}^{r} \alpha_{i k} h_{k p}\right) \alpha_{i j}+\left(\sum_{k=I}^{r} \beta_{i k} h_{k p}\right) \beta_{i j}\right]=\lambda h_{j p}
$$

Thus, the column vector $\boldsymbol{h}_{p}$ is obtained from eq.(7) for $j=1,2, \ldots, r$.

Now, introducing a matrix $\Gamma_{p}$, the pseudo diagonalization is resulted in the eigenvalue problem. The matrix $\Gamma_{p} \in R^{r x r}$ is defined as

$$
\begin{array}{r}
\Gamma_{p} \equiv\left(\gamma_{k j}{ }^{p}\right), \quad \gamma_{k j}{ }^{p} \equiv \sum_{i=1, i \neq p}^{m}\left(\alpha_{i k} \alpha_{i j}+\beta_{i k} \beta_{i j}\right) \\
(k, j=1,2, \ldots, r)
\end{array}
$$

Calculating the $j$ th element of $\Gamma_{p} \boldsymbol{h}_{p}$, we get

$$
\begin{aligned}
& \gamma_{j 1}{ }^{p} h_{l p}+\gamma_{j 2}{ }^{p} h_{2 p}+\cdots+\gamma_{j r}{ }^{p} h_{r p} \\
& =\left(\sum_{k=1}^{r} \alpha_{l k} h_{k p}\right) \alpha_{l j}+\cdots+\left(\sum_{k=1}^{r} \alpha_{p-l, k} h_{k p}\right) \alpha_{p-l j} \\
& +\left(\sum_{k=1}^{r} \alpha_{p+l k} h_{k p}\right) \alpha_{p+l j}+\cdots+\left(\sum_{k=1}^{r} \alpha_{m k} h_{k p}\right) \alpha_{m j} \\
& +\left(\sum_{k=1}^{r} \beta_{1 k} h_{k p}\right) \beta_{1 j}+\cdots+\left(\sum_{k=1}^{r} \beta_{p-1, k} h_{k p}\right) \beta_{p-1 j} \\
& +\left(\sum_{k=1}^{r} \beta_{p+1, k} h_{k p}\right) \beta_{p+l j}+\cdots+\left(\sum_{k=1}^{r} \beta_{m k} h_{k p}\right) \beta_{m j} \\
& =\sum_{i=l, i \neq p}^{m}\left[\left(\sum_{k=1}^{r} \alpha_{i k} h_{k p}\right) \alpha_{i j}\right]+\sum_{i=l, i \neq p}^{m}\left[\left(\sum_{k=1}^{r} \beta_{i k} h_{k p}\right) \beta_{i j}\right]
\end{aligned}
$$

By comparing with eq.(7), we see that eq. (9) equals to $\lambda h_{j p}$ for $j=1,2, \ldots, r$. Thus, the next relation holds.

$$
\Gamma_{p} \boldsymbol{h}_{p}=\lambda \boldsymbol{h}_{p}
$$

Furthermore, calculating $\boldsymbol{h}_{p}{ }^{T} \Gamma_{p} \boldsymbol{h}_{p}$, we get

$$
\begin{aligned}
& \boldsymbol{h}_{p}{ }^{T} \Gamma_{p} \boldsymbol{h}_{p}=\left[h_{l p}, h_{2 p}, \cdots, h_{r p}\right]\left[\begin{array}{cccc}
\gamma_{1 I}{ }^{p} & \gamma_{12}{ }^{p} & \cdots & \gamma_{l r}{ }^{p} \\
\gamma_{2 l}{ }^{p} & \gamma_{22}{ }^{p} & \cdots & \gamma_{2 r}{ }^{p} \\
\vdots & \vdots & \ddots & \vdots \\
\gamma_{r l}{ }^{p} & \gamma_{r 2}{ }^{p} & \cdots & \gamma_{r r}{ }^{p}
\end{array}\right]\left[\begin{array}{c}
h_{l p} \\
h_{2 p} \\
\vdots \\
h_{r p}
\end{array}\right] \\
& \quad=\left(\sum_{k=1}^{r} \alpha_{1 k} h_{k p}\right)^{2}+\cdots+\left(\sum_{k=1}^{r} \alpha_{p-1, k} h_{k p}\right)^{2} \\
& +\left(\sum_{k=1}^{r} \alpha_{p+1, k} h_{k p}\right)^{2}+\cdots+\left(\sum_{k=1}^{r} \alpha_{m k} h_{k p}\right)^{2} \\
& +\left(\sum_{k=1}^{r} \beta_{I k} h_{k p}\right)^{2}+\cdots+\left(\sum_{k=1}^{r} \beta_{p-1, k} h_{k p}\right)^{2} \\
& +\left(\sum_{k=1}^{r} \beta_{p+1, k} h_{k p}\right)^{2}+\cdots+\left(\sum_{k=1}^{r} \beta_{m k} h_{k p}\right)^{2} \\
& \quad=\sum_{i=1, i \neq p}^{m}\left[\left(\sum_{k=1}^{r} \alpha_{i k} h_{k p}\right)^{2}+\left(\sum_{k=1}^{r} \beta_{i k} h_{k p}\right)^{2}\right]
\end{aligned}
$$

By comparing eq.(11) with eqs.(3), (5), and (6), the next relation also holds.

$$
\boldsymbol{h}_{p}{ }^{T} \Gamma_{p} \boldsymbol{h}_{p}=\sum_{i=l, i \neq p}^{m}\left|\ell_{i p}\left(j \omega_{0}\right)\right|^{2}
$$

Substituting eq.(10) into the left side of eq.(12), and in view of eq.(4), we have

$$
\boldsymbol{h}_{p}{ }^{T} \Gamma_{p} \boldsymbol{h}_{p}=\lambda \boldsymbol{h}_{p}{ }^{T} \boldsymbol{h}_{p}=\lambda\left\|\boldsymbol{h}_{p}\right\|^{2}=\lambda
$$

These mean that the magnitude of the interaction, eq.(3), corresponds to the eigenvalue of the matrix $\Gamma_{p}$, 
and then $\lambda$ must be a minimum eigenvalue from property of the problem. Therefore, the $p$ th column vector $\boldsymbol{h}_{p}$ of the precompensator $G c$ is the eigenvector of the minimum eigenvalue $\lambda$ for the matrix $\Gamma p$. After all, the design of the precompensator $G c$ results in the eigenvalue problem of the matrices $\Gamma_{1}, \Gamma_{2}, \ldots, \Gamma_{m}$. The result obtained is in agreement with the result of the case, the numbers of input and output are equal.

\section{Simulation}

\subsection{A three inputs - two outputs controlled system}

Let us consider a three inputs - two outputs controlled system eq.(14) as an example to investigate the effectiveness of the proposed method. This case corresponds to $r>m$.

$$
\begin{aligned}
& G p_{I 1}=\frac{1}{s+1} e^{-4 s} \cong \frac{s^{2}-1.5 s+0.75}{s^{3}+2.5 s^{2}+2.25 s+0.75} \\
& G p_{21}=\frac{2}{s+1} e^{-8 s} \cong \frac{2 s^{2}-1.5 s+0.375}{s^{3}+1.75 s^{2}+0.9375 s+0.1875} \\
& G p_{I 2}=\frac{2}{s+3} e^{-20 s} \cong \frac{2 s^{2}-0.6 s+0.06}{s^{3}+3.3 s^{2}+0.93 s+0.09} \\
& G p_{22}=\frac{3}{s+4} e^{-24 s} \cong \frac{3 s^{2}-0.75 s+0.0625}{s^{3}+4.25 s^{2}+1.021 s+0.08333} \\
& G p_{13}=\frac{2}{s+4} e^{-10 s} \cong \frac{2 s^{2}-1.2 s+0.24}{s^{3}+4.6 s^{2}+2.52 s+0.48} \\
& G p_{23}=\frac{2}{s+2} e^{-15 s} \cong \frac{s^{2}-0.8 s+0.1067}{s^{3}+2.4 s^{2}+0.8533 s+0.1067}
\end{aligned}
$$

For the transfer function matrix $G p(s)$ of eq.(14), the precompensator $G c$ is designed at frequency $\omega_{c l}=1.0$ in the first place. This frequency $\omega_{c l}$ is a kind of hunting frequency for the plant, which has very strong interaction among controlled variables, and then frequency $\omega_{c l}$ is selected by the preparatory experiment. Otherwise, $\omega_{c l}$ is chosen suitably, because the precompensator can be improved using another frequency $\omega_{c i}$ and precompensators connected in series or parallel in the next place [5]. The precompensator $G c$ is designed at $\omega_{c l}=1.0$ as

$$
G_{c}=\left[\begin{array}{rr}
-0.0819 & -0.3376 \\
-0.7116 & -0.5319 \\
0.3978 & 0.4888
\end{array}\right]
$$

For evaluation of the interaction, a diagonal ( row or column ) dominance is used in general. The diagonal dominance of the inverse matrix $Q^{\wedge}(s)=\left[q^{\wedge} i j(s)\right]$ of a transfer function matrix $Q(s)$ on the imaginary axis is equivalent to that one side of the inequalities is valid for all $\omega$,

$$
\begin{aligned}
& \left|\hat{q}_{i i}(j \omega)\right|>d_{i}(j \omega)=\sum_{j=l, j \neq i}^{m}\left|\hat{q}_{i j}(j \omega)\right|, i=1,2, \ldots, m \\
& \left|\hat{q}_{i i}(j \omega)\right|>d_{i}{ }^{\prime}(j \omega)=\sum_{j=l, j \neq i}^{m}\left|\hat{q}_{j i}(j \omega)\right|, i=1,2, \ldots, m
\end{aligned}
$$

The inequalities (16.a) and (16.b) mean that the circle with its center at $q^{\wedge} i i(j \omega)$ and with a radius of $d_{i}(j \omega)$ or $d_{i}^{\prime}(j \omega)$ does not include the origin. The set of vector loci is the inverse Nyquist array, and $d_{i}(j \omega)$ and $d_{i}{ }^{\prime}(j \omega)$ are Gershgorin band, and then, the Gershgorin bands show a degree of the interaction for the controlled system.

The block diagram, inverse Nyquist diagrams and Gershgorin bands of the transfer function matrix $G(s)=$ $G p(s) G c$ are illustrated in Fig.2 and Fig.3, respectively. In this case, the Gershgorin bands include the origin, and then the diagonal dominance does not succeed.

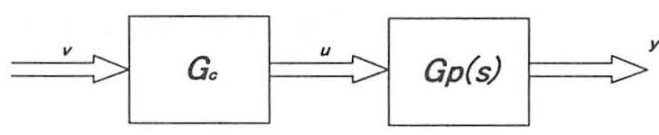

Fig.2. Block diagram with precompensator $G c$ connected in series

When we use one precompensator only, it is difficult to realize the diagonal dominance for the controlled system with very strong interaction. Therefore, a new precompensator $G_{c 2}$ is connected in series as shown in Fig.4, and designed at frequency $\omega_{c 2}=6.0$, which is the frequency of strong interaction, regarding the transfer function matrix $G p(s) G_{c l}$ as a new controlled system $G p(s)^{\prime}=G p(s) G_{c l}$. 

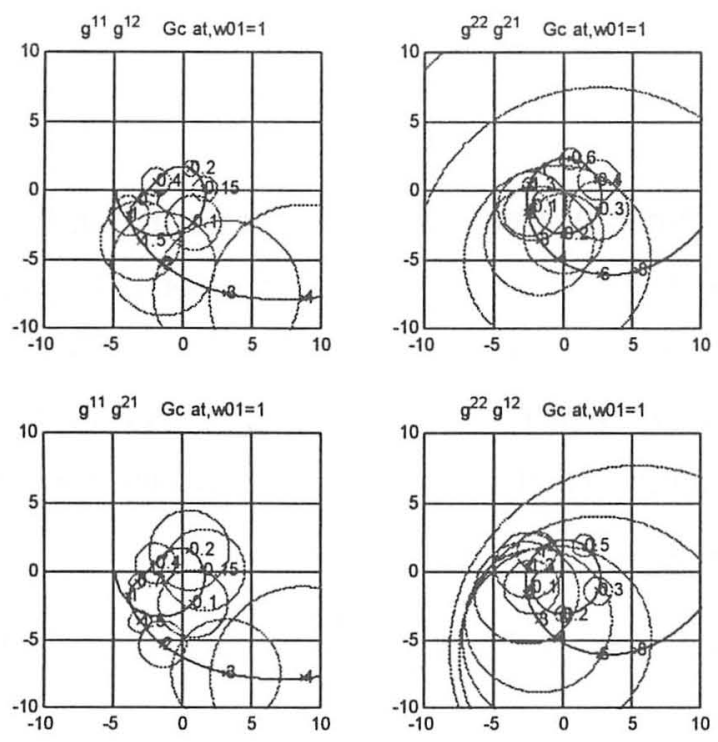

Fig.3. Inverse Nyquist array of $G(s)=G p(s) G c$

Then the second stage precompensator $G c=G_{c l} G_{c 2}$ is designed as

$$
G_{c}=G_{c 1} \times G_{c 2}=\left[\begin{array}{rr}
0.1097 & 0.1056 \\
-0.3210 & -0.3312 \\
0.3321 & 0.3417
\end{array}\right]
$$

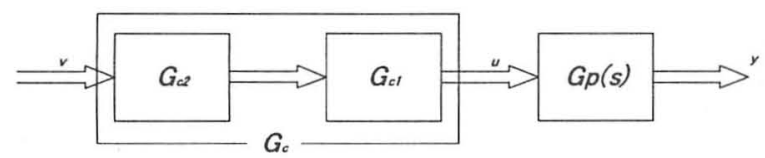

Fig.4. Second stage series connection of precompensator $G_{c 2}$

The inverse Nyquist diagrams and Gershgorin bands of $G p(s) G_{c 2} G_{c l}$ are shown in Fig.5, and then at least the diagonal column dominance, which is indicated by the graphs of $g^{11}-g^{21}$ and $g^{22}-g^{12}$, is realized at every frequency.

Using the precompensator (17), PI controller as the main controller $K(s)$ of the closed loop system shown in Fig.1 is designed for each loop of the controlled variables by the ultimate sensitivity method, and then the control result is illustrated in Fig.6.

\subsection{A two inputs - three outputs controlled system}

Next, consider a two inputs - three outputs controlled system eq.(18), which transfer functions are deduced from step responses of an experimental plant [5].
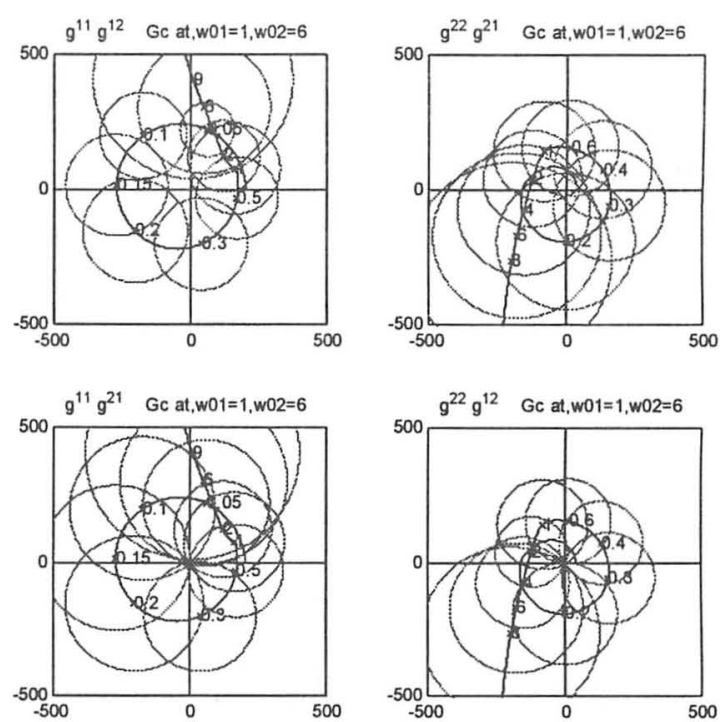

Fig.5. Inverse Nyquist array by $G_{c 2}$ connected in series

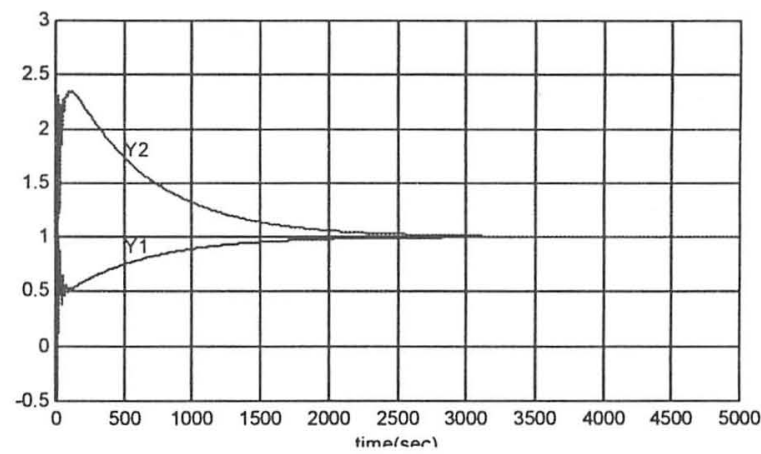

Fig.6. Noninteracting PI control with precompensators connected in series

$$
\begin{aligned}
G p_{11}= & \frac{37}{114.29 s+1} e^{-189.29 s} \\
& \cong \frac{37 s^{2}-1.173 s+0.01239}{114.3 s^{3}+4.623 s^{2}+0.06998 s+0.0003349} \\
G p_{21}= & \frac{30}{100 s+1} e^{-6.26 s} \\
& \cong \frac{30 s^{2}-28.8 s+9.219}{100 s^{3}+97 s^{2}+31.68 s+0.3072} \\
G p_{31}= & \frac{50}{130 s+1} e^{-20 s} \\
& \cong \frac{50 s^{2}-15 s+1.5}{130 s^{3}+40 s^{2}+4.2 s+0.03} \\
G p_{12}= & \frac{17.41}{334.69 s+1} e^{-40.82 s} \\
& \cong \frac{17.41 s^{2}-2.559 s+0.1254}{334.7 s^{3}+50.2 s^{2}+2.558 s+0.007203} \\
G p_{22}= & \frac{40}{140 s+1} e^{-30 s}
\end{aligned}
$$




$$
\begin{aligned}
& \cong \frac{40 s^{2}-8 s+0.5333}{140 s^{3}+29 s^{2}+2.067 s+0.01333} \\
G p_{32}=\frac{59.31}{122.73 s} e^{-7.73 s} & \cong \frac{59.31 s^{2}-46.05 s+11.92}{122.7 s^{3}+88.53 s^{2}+23.45 s+0.201}
\end{aligned}
$$

For the transfer function matrix $G p(s)$ of eq.(18), the precompensator $G c$ is designed at $\omega_{c l}=0.013$, which is the hunting frequency when a control method is carried out for the experimental plant.

The precompensator $G c$ is designed as

$$
G_{c}=\left[\begin{array}{rrr}
0.7825 & -0.4612 & 0.8023 \\
-0.6227 & 0.8873 & -0.5970
\end{array}\right]
$$

In this case of $r<m$, the inverse matrix $[G p(s) G c]^{-l}$ does not exist for any matrix $G c$ in general (this reason will be described at another opportunity). Therefore, the Nyquist diagram and its Gershgorin band are used to evaluate the diagonal dominance in this case. The block diagram and results are illustrated in Fig.7 and Fig.8, and the Gershgorin bands include the origin, then the diagonal dominance does not succeed.

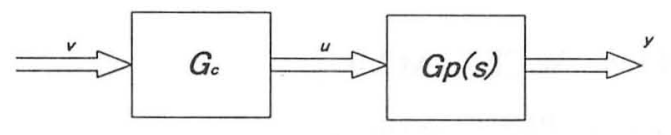

Fig.7. Precompensator $G c$ connected in series
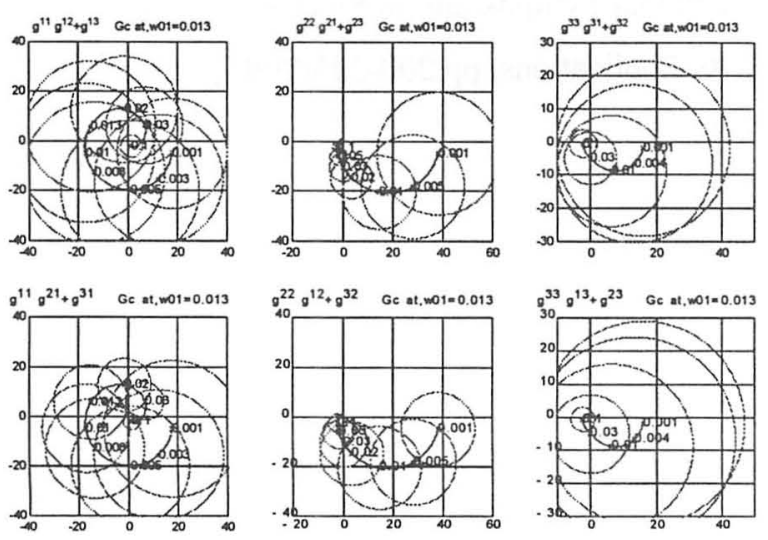

Fig.8. Nyquist array by $G c$

A second stage precompensator $G_{c 2}$ is connected in series here as shown in Fig.9. The precompensator Gc

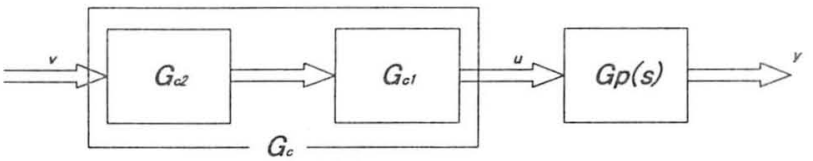

Fig.9. Second stage series connection of $G_{c 2}$ $=G_{c l} G_{c 2}$ is designed as

$$
G_{c}=G_{c 1} \times G_{c 2}=\left[\begin{array}{rrr}
0.8758 & 1.1273 & -0.4057 \\
-0.4793 & -1.3796 & 0.6012
\end{array}\right]
$$

and the Nyquist diagrams and Gershgorin bands are illustrated in Fig.10.
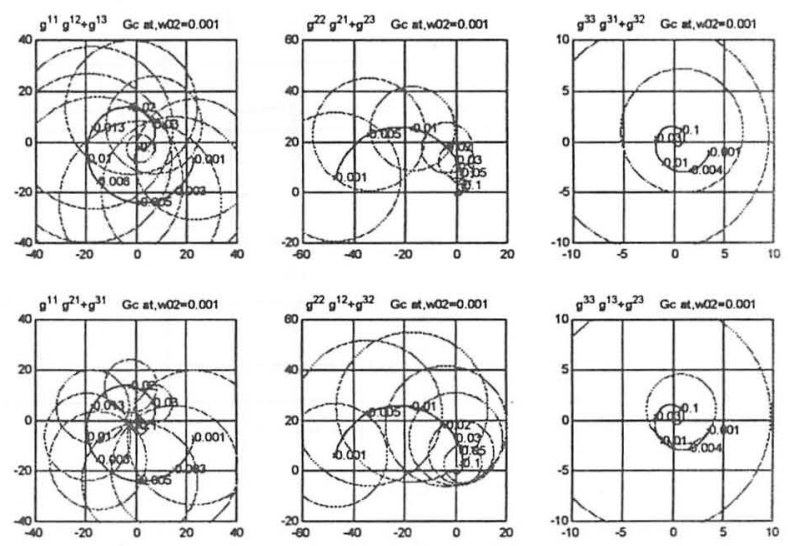

Fig.10. Nyquist array by $G_{c l} G_{c 2}$

Furthermore, a third stage precompensator $G_{c 3}$ is connected as shown in Fig.11. In this case, precompensator $G c=G_{c 1} G_{c 2} G_{c 3}$ is as

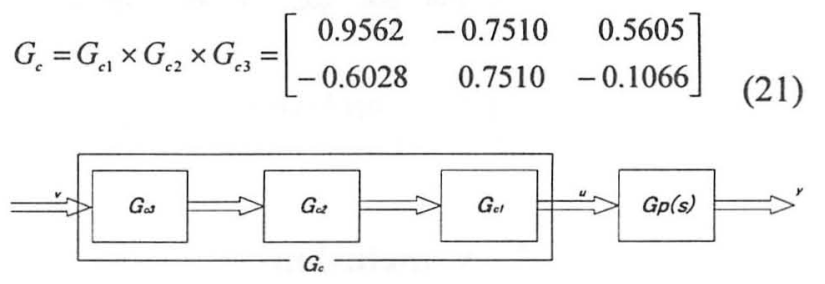

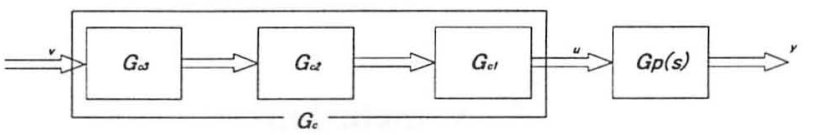

Fig.11. Three stage series connection of $G_{c 3}$

and Nyquist diagrams and Gershgorin bands are illustrated in Fig.12. In this case, the diagonal column dominance $\left(g^{11}-g^{21} g^{31}, g^{22}-g^{12} g^{32}\right.$, and $\left.g^{33}-g^{13} g^{23}\right)$ holds relatively on comparing Fig. 8 and Fig.10. The control results are illustrated in Fig.13 using the precompensator (21). As the result, we can confirm the effectiveness of the proposed method. 

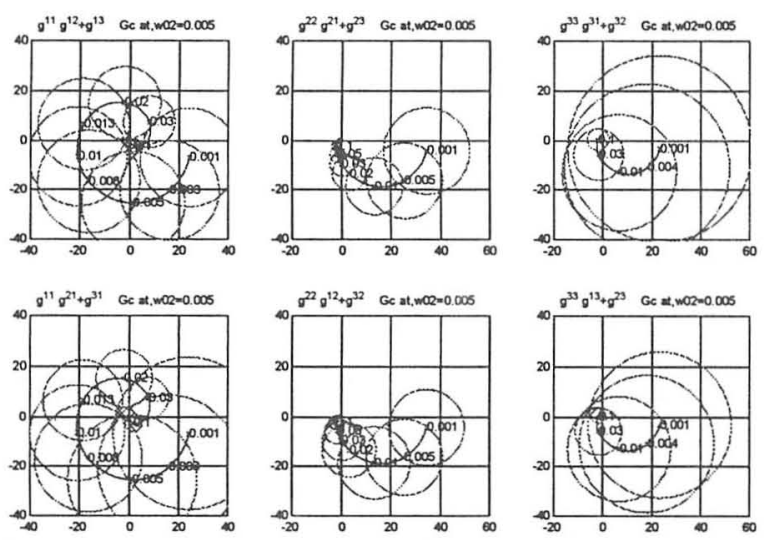

Fig.12. Nyquist array by $G_{c l} G_{c 2} G_{c 3}$

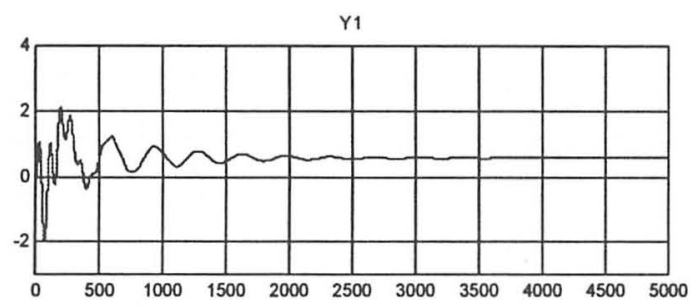

Y2

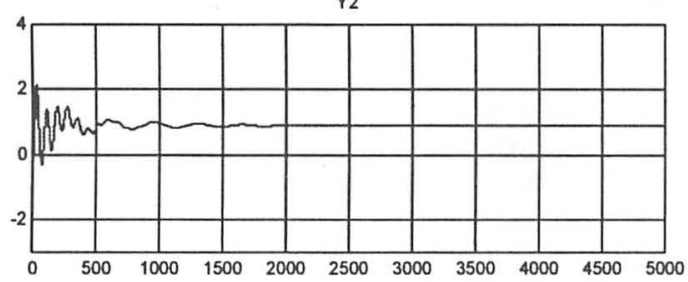

Y3

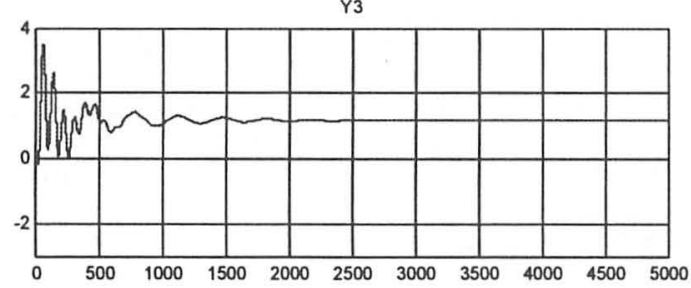

Fig.13. Noninteracting PI control with precompensators connected in series

\section{Conclusion}

This paper proposed generalization of the pseudo diagonalization method. The inverse Nyquist array method is one of the practical design methods for multi-input and multi-output systems, and the design of the noninteracting precompensator is reduced to the eigenvalue problem by the pseudo diagonalization method. However, the pseudo diagonalization method was applicable only to the system where numbers of input and output are equal. By the generalized method proposed, the pseudo diagonalization could be applicable to the system where numbers of input and output are not always equal. The indication of the effectiveness of the proposed method was given by some simulations.

\section{References}

[1] H.H.Rosenbrock: Design of Multivariable Control Systems using the Inverse Nyquist Array, Proc.IEE, Vol.115(1969)

[2] P.L.Falb and W.A.Wolovich: Decoupling in the Design and Synthesis of Multivariable Control Systems, IEEE, AC-12(1967)

[3] D.J.Hawkins: Pseudo Diagonalization and the Inverse Nyquist array method, Proc.IEE, Vol.119, No.3, pp.337-342(1972)

[4] M.Ito, H.Kimura, S.Hosoe: Theory of Design for Linear Control Systems, SICE(1978)

[5] H.Lee, W.Choi, M.Miyazaki and K.Akizuki: Noninteracting PID Control using Precompensators with Series-Parallel Connections, Proc. of the 33rd International Symposium on Stochastic Systems Theory and Its Applications, pp.200-205(2001) 